\title{
Empowerment, vulnerabilidad al estrés $y$ burnout en enfermeros portugueses
}

\author{
Empowerment, stress vulnerability and burnout \\ among Portuguese nursing staff
}

Alejandro Orgambídez-Ramos ${ }^{1}$

Yolanda Borrego-Alés ${ }^{2}$

Carlos Ruiz-Frutos ${ }^{2}$

${ }^{1}$ Research Centre for Spatial and Organizacional Dynamics, Universidade do Algarve. Estr. da Penha 139. 8005-139. Faro Portugal. aoramos@ualg.pt

${ }^{2}$ Universidad de Huelva.

Huelva Espanha.

\begin{abstract}
The work environment in Portuguese hospitals, characterized by economic cutbacks, can lead to higher levels of burnout experienced by nursing staff. Furthermore, vulnerability to stress can negatively affect the perception of burnout in the workplace. However, structural empowerment is an organizational process that can prevent and decrease burnout among nurses. Consequently, the aim of the study was to examine to what extent structural empowerment and vulnerability to stress can play a predictive role in core burnout in a sample of Portuguese nurses. A convenience sample of 297 nursing staff members from Portuguese hospitals was used in this study. Core burnout was negatively and significantly related to all the dimensions of structural empowerment, and it was positively and significantly related to vulnerability to stress. Regression models showed that core burnout was significantly predicted by access to funds, access to opportunities and vulnerability to stress. Organizational administrations must make every effort in designing interventions focused on structural empowerment, as well as interventions focused on individual interventions that enhance skills for coping with stress.
\end{abstract}

Key words Empowerment, Professional burnout, Personality, Nursing staff
Resumen El contexto laboral en los hospitales portugueses, caracterizado por los recortes económicos, puede contribuir al aumento de los niveles de burnout experimentados por el personal de enfermería. Además, la vulnerabilidad al estrés en estos profesionales afecta negativamente a la percepción del estrés en el contexto de trabajo. No obstante, el empowerment estructural es un proceso organizacional que puede prevenir y aliviar el burnout en este colectivo profesional. En este sentido, el objetivo de este estudio es comprobar el papel predictor del empowerment estructural y la vulnerabilidad al estrés sobre el burnout en una muestra de enfermeros portugueses. Se utilizó una muestra por conveniencia de 297 profesionales enfermeros de hospitales de Portugal. El core burnout se relacionó negativa y significativamente con todas las dimensiones del empowerment estructural, y positivamente con la vulnerabilidad al estrés. Los modelos de regresión lineal múltiple mostraron como predictores significativos del core burnout el acceso a recursos, el acceso a oportunidades y la vulnerabilidad al estrés. Las administraciones deben diseñar intervenciones centradas en el empowerment estructural, pero también en intervenciones individuales que mejoren las capacidades de afrontamiento del estrés.

Palabras clave Empowerment, Agotamiento profesional, Personalidad, Personal de enfermería 


\section{Introducción}

En los últimos años la crisis económica ha impuesto duros recortes económicos en la Unión Europea en general y en Portugal en particular. Concretamente, los hospitales y las unidades de gestión de salud han sido uno de los objetivos de dichas medidas. En consecuencia, el colectivo de profesionales de enfermería está haciendo frente a mayores demandas en la atención y cuidado de la salud con menores recursos, creando contextos y situaciones laborales de riesgo psicosocial que pueden conducir a experiencias de burnout ${ }^{1}$. Por otro lado, determinados rasgos de personalidad, tales como la extraversión o la vulnerabilidad al estrés, pueden afectar a la percepción de los niveles de estrés y burnout en el puesto de trabajo².

En este contexto, aparece el empowerment estructural como una herramienta organizacional que permite la optimización de las competencias personales y profesionales, a la par que incrementa los niveles de satisfacción y bienestar en los trabajadores ${ }^{3}$. De acuerdo con la teoría de Kanter ${ }^{4}$ sobre empowerment estructural, las características formales e informales de las organizaciones determinan los niveles de empowerment de sus miembros. Según esta autora, los trabajadores son potenciados (empowered) cuando su entorno de trabajo les proporciona oportunidades para el crecimiento y el acceso al "poder" necesario para cumplir con las exigencias del trabajo.

Varios estudios han mostrado las relaciones entre empowerment estructural y desempeño, rendimiento, satisfacción laboral y estrés en profesionales de enfermería ${ }^{1,5-7}$. No obstante, apenas existen estudios realizados en unidades de salud y personal de enfermería en Portugal que analicen las relaciones entre el empowerment estructural, la vulnerabilidad al estrés y el burnout. En este sentido, el objetivo de este estudio es comprobar el papel predictor del empowerment estructural y de la vulnerabilidad al estrés, como rasgo de personalidad, sobre los niveles de burnout en una muestra de profesionales de enfermería portugueses.

\section{Marco teórico}

El concepto de empowerment es ampliamente conocido tanto por los investigadores como por los profesionales de los recursos humanos, lo que ha conllevado la aparición de numerosas escalas para evaluarlo e intervenir en las unidades de salud $^{8}$. No obstante, solo Kanter ${ }^{4}$ ofrece un modelo teórico fundamentado empíricamente sobre los elementos estructurales del contexto de trabajo que facilitan el empowerment de los profesionales: el empowerment estructural.

De acuerdo con Kanter ${ }^{4}$, las características del ambiente de trabajo determinan las actitudes y comportamientos laborales, independientemente de las predisposiciones personales o rasgos de personalidad. Esta autora expresa que los trabajadores se sienten empowered cuando su entorno de trabajo les proporciona oportunidades para el crecimiento y el acceso al "poder" necesario para cumplir los objetivos del trabajo. El "poder" se define como la capacidad para movilizar recursos y lograr objetivos ${ }^{4}$, de forma que cuando existen recursos y oportunidades en el contexto de trabajo, los trabajadores poseen "poder" para poder realizar sus tareas ${ }^{5,9}$.

Kanter ${ }^{4}$ diferencia dos tipos de poder: poder formal y poder informal. El primero se relaciona con características del trabajo y de la organización tales como flexibilidad, adaptabilidad, innovación y toma de decisiones autónoma, junto con el impacto y la visibilidad en el funcionamiento de la empresa. El segundo está asociado a las redes sociales y a la comunicación dentro de la organización, encuadradas por las relaciones con los compañeros, superiores y otros miembros. Además, estos dos tipos de poder facilitan el acceso a otros elementos organizacionales ${ }^{5,9,10}$ : acceso a oportunidades, a la información, al apoyo y a los recursos en el entorno laboral.

$\mathrm{El}$ acceso a las oportunidades se refiere a las oportunidades de crecimiento y promoción en la empresa, así como oportunidades de adquirir conocimientos y competencias. Específicamente, Kanter ${ }^{4}$ hace referencia a las posibilidades de avanzar en la organización, mediante la exposición y visibilidad profesional, junto con la habilidad de aprender y desarrollarse profesionalmente en el puesto de trabajo. El acceso a la información hace referencia a los conocimientos, de tipo tecnológico y de experto, necesarios para ser efectivo y cumplir con las tareas en el trabajo. El acceso al apoyo consiste en recibir feedback, orientación y consejo por parte de subordinados, compañeros y superiores para tomar decisiones en el contexto organizacional. Finalmente, el acceso a los recursos hace referencia a los materiales, medios y tiempo para realizar el trabajo ${ }^{5,9,10}$.

Diversos estudios han observado una fuerte relación positiva entre el empowerment estructural y actitudes laborales, tales como la satisfacción o el compromiso organizacional ${ }^{3}$, y una fuerte relación negativa con el estrés y el bur- 
nout $^{7,11,12}$. El burnout en profesionales de enfermería no solo se manifiesta en el cansancio físico o el agotamiento emocional, sino que afecta especialmente al trato con los pacientes y a la calidad del cuidado y la atención ${ }^{7}$.

Además de las características del entorno del trabajo señaladas por Kanter ${ }^{4}$, también existe evidencia científica de que ciertos rasgos de la personalidad afectan a cómo las personas afrontan las situaciones de estrés en el trabajo ${ }^{13}$. Entre estos rasgos se destacan especialmente la personalidad Tipo A, los rasgos de personalidad del modelo Big Five (Neuroticismo, Extraversión, Apertura a la Experiencia, Amabilidad y Responsabilidad) y la vulnerabilidad al estrés ${ }^{2,14,15}$.

La personalidad Tipo A (competitividad, estilo de vida apresurado, necesidad de control) se ha asociado con elevados niveles de burnout ${ }^{14}$. De manera similar, se ha observado que determinados rasgos de personalidad influyen en el tipo de estrategia de afrontamiento del estrés ${ }^{16}$. El Neuroticismo parece ser el predictor más potente del burnout de los cinco rasgos del Big Five ${ }^{2,17,18}$. Relacionado con el Neuroticismo, la vulnerabilidad al estrés también ha sido relacionado con el burnout experimentado en enfermería ${ }^{15}$. Los individuos más vulnerables suelen ser más sensibles al estrés, sintiéndose sobrecargados en situaciones de trabajos cotidianas. En consecuencia, son más propensos a experimentar burnout incluso en situaciones consideradas como normales por el resto de las personas ${ }^{15,19,20}$.

Varios estudios han registrado relaciones entre el empowerment estructural y el burnout en profesionales de enfermería ${ }^{1,3,5,21}$. No obstante, la investigación sobre las relaciones entre empowerment estructural, vulnerabilidad al estrés y burnout es escasa y prácticamente no se ha llevado a cabo en el contexto de salud de Portugal. En este sentido, el objetivo de este estudio es comprobar el papel predictor del empowerment estructural y la vulnerabilidad al estrés sobre el burnout en una muestra de enfermeros y enfermeras del sur de Portugal.

\section{Método}

\section{Diseño y muestra}

Se llevó a cabo un estudio transversal, descriptivo y correlacional mediante el uso de cuestionarios $^{22}$. Un total de 478 profesionales enfermeros de tres hospitales públicos del sur de Portugal fueron encuestados, obteniéndose una muestra final de 297 encuestados $(62.13 \%$ de tasa de respuesta). 189 participantes (63.6\%) fueron mujeres, siendo la edad media de la muestra de 37.42 años. La mayoría de los participantes expresó estar trabajando a turnos.

\section{Medidas}

Core burnout. El core burnout fue evaluado con la versión portuguesa ${ }^{23}$ del Maslach Burnout Inventory $(\mathrm{MBI})^{24}$, utilizando para ello las dimensiones de agotamiento (8 ítems) y despersonalización (4 ítems). Las respuestas fueron dadas en una escala Likert de 1: Nunca a 7: Cada día. Elevadas puntuaciones indican elevados niveles de burnout presentes en los participantes. El coeficiente alpha de Cronbach obtenido en este estudio fue de .85 .

Empowerment estructural. Se utilizó la versión portuguesa ${ }^{25}$ de la escala Conditions for Work Effectiveness Questionnaire (CWEQ-II) de Laschinger et al. ${ }^{9}$ para medir el empowerment estructural. Los 19 ítems de la escala se agrupan en las 6 dimensiones del empowerment indicadas por Kanter ${ }^{4}$ : oportunidades ( 3 ítems), información (3 ítems), apoyo (3 ítems), recursos (3 ítems), poder formal (4 ítems) y poder informal (5 ítems). Las respuestas son dadas mediante una escala tipo Likert de 1: Nunca a 5: Mucho. Altas puntuaciones expresan elevados niveles de empowerment estructural presentes en los participantes. Los coeficientes de fiabilidad (alpha de Cronbach) de las dimensiones fueron aceptables, desde .77 a .88 .

Vulnerabilidad al estrés. Para medir la vulnerabilidad al estrés se utilizó la escala 23 QVS de Serra ${ }^{20}$. Esta escala consta de 23 ítems que se responden a través de una escala tipo Likert de 5 puntos, desde 1: Totalmente en desacuerdo a 5: Totalmente de acuerdo. Elevadas puntuaciones indican altos niveles de vulnerabilidad al estrés en los participantes. El coeficiente alpha de Cronbach obtenido en este estudio fue de .78 .

\section{Recogida de datos}

Los cuestionarios fueron aplicados entre febrero y mayo de 2013. Tras el pedido de autorización, todos los comités éticos de los hospitales aprobaron el estudio. Los cuestionarios fueron aplicados individualmente o en pequeños grupos al inicio del turno laboral, proporcionando todo el tiempo que los participantes necesitaran. La participación fue voluntaria y en todo momento 
se garantizó la confidencialidad y anonimato de todos los datos.

\section{Análisis de datos}

El análisis de datos fue llevado a cabo con el paquete estadístico STATA v13. Se calcularon los estadísticos descriptivos de las variables (media, desviación típica, asimetría), así como las correlaciones entre ellas y los coeficientes de fiabilidad (alpha de Cronbach) de las escalas. Modelos de regresión lineal múltiple fueron realizados para comprobar el papel predictor del empowerment estructural y de la vulnerabilidad al estrés sobre el core burnout.

\section{Resultados}

Estadísticos descriptivos y correlaciones. En la Tabla 1 se muestran la media, desviación típica, alpha de Cronbach y correlaciones de las variables del estudio. De forma general, los profesionales enfermeros no percibieron su lugar de trabajo como especialmente motivante en términos de empowerment. Las medias en las dimensiones del empowerment estructural fueron desde 3.86 (acceso a oportunidades) a 2.90 (acceso a recursos). Los participantes experimentaron mayores niveles de poder informal $(\mathrm{M}=3.60, \mathrm{DT}=0.71)$ que de poder formal $(\mathrm{M}=2.66$, $\mathrm{DT}=0.79)$. En relación con el core burnout y la vulnerabilidad al estrés, los profesionales enfermeros mostraron niveles bajos en ambas variables $(\mathrm{M}=1.56$, DT $=0.95)$ y M $=1.51, \mathrm{DT}=0.34$, respectivamente).
Como fue esperado, todas las dimensiones del empowerment estructural y los dos tipos de poder, formal e informal, se relacionaron negativamente con el core burnout, con valores de coeficientes de Pearson desde -.36 a -.19 (p < $0,001)$. Mayores puntuaciones en las dimensiones del empowerment estructural se asociaron a menores puntuaciones en core burnout. Por el contrario, se observó una relación positiva y significativa entre la vulnerabilidad al estrés y el core burnout $(\mathrm{r}=.37, \mathrm{p}<0,001)$.

Modelos de regresión lineal múltiple. Para comprobar el objetivo del estudio, se llevaron a cabo varios modelos de regresión lineal múltiple y jerárquica. Estos modelos se utilizaron para verificar el papel predictor del empowerment estructural (acceso a oportunidades, información, apoyo y recursos, poder formal e informal) y la vulnerabilidad al estrés sobre los niveles de core burnout en los participantes. Antes del análisis, se verificó que los supuestos de normalidad, multicolinearidad y homoscedasticidad no se violaron.

En primer lugar, considerando la teoría de Kanter relativa al empowerment estructural, en el primer paso (M1) se incluyeron el poder formal y el poder informal como predictores del core burnout en el modelo jerárquico de regresión. El total de varianza explicada por el modelo fue de $13.70 \%(\mathrm{~F}(2,294)=23.34, \mathrm{p}<0,001)$. De los dos tipos de poder, el poder informal se mostró como un predictor significativo $(\mathrm{p}<0,001)$ con un valor beta de -.32 (Tabla 2).

Tras incluir las variables acceso a oportunidades, información, apoyo y recursos en el segundo paso del modelo (M2), la varianza total explica-

Tabla 1. Estadísticos descriptivos y correlaciones de todas las variables del estudio.

\begin{tabular}{|c|c|c|c|c|c|c|c|c|}
\hline & 1 & 2 & 3 & 4 & 5 & 6 & 7 & 8 \\
\hline 1. Core burnout & 1 & $0,37^{* *}$ & $-0,28^{* *}$ & $-0,34^{* *}$ & $-0,19^{* *}$ & $-0,36^{* *}$ & $-0,24^{* *}$ & $-0,36^{* *}$ \\
\hline 2. Vulner. estrés & & 1 & $-0,13$ & $-0,19^{*}$ & $-0,01$ & 0,01 & $-0,04$ & $-0,22^{* *}$ \\
\hline 3. Oportunidades & & & 1 & $0,36^{* *}$ & $0,21^{* *}$ & $0,16^{*}$ & $0,28^{* *}$ & $0,41^{* *}$ \\
\hline 4. Información & & & & 1 & $0,35^{* *}$ & $0,42^{* *}$ & $0,41^{* *}$ & $0,45^{* *}$ \\
\hline 5. Ароуо & & & & & 1 & $0,30^{* *}$ & $0,45^{* *}$ & $0,38^{* *}$ \\
\hline 6. Recursos & & & & & & 1 & $0,47^{* *}$ & $0,42^{* *}$ \\
\hline 7. Poder formal & & & & & & & 1 & $0,42^{* *}$ \\
\hline 8. Poder informal & & & & & & & & 1 \\
\hline Media & 1,57 & 1,51 & 3,86 & 3,52 & 3,06 & 2,90 & 2,66 & 3,60 \\
\hline Desviación típica & 0,95 & 0,43 & 0,80 & 0,75 & 0,89 & 0,72 & 0,79 & 0,71 \\
\hline Asimetría & 0,97 & 0,07 & $-0,47$ & $-0,27$ & $-0,13$ & 0,05 & $-0,04$ & $-0,24$ \\
\hline Alpha de Cronbach & 0,85 & 0,78 & 0,84 & 0,88 & 0,86 & 0,77 & 0,78 & 0,78 \\
\hline
\end{tabular}

${ }^{\star} \mathrm{p}<.05^{\star *} \mathrm{p}<.01$. 
Tabla 2. Modelos de regresión jerárquica sobre core burnout.

\begin{tabular}{lrrrrr}
\hline & B & SE B & $\mathbf{t}$ & $\mathbf{p}>|\mathbf{t}|$ & Beta \\
\hline M1 & & & & & \\
Cte & 3,40 & 0,27 & 12,43 & 0,000 & --- \\
Poder formal & $-0,11$ & 0,07 & $-1,56$ & 0,119 & $-0,09$ \\
Poder informal & $-0,43$ & 0,08 & $-5,27$ & 0,000 & $-0,32$ \\
M2 & & & & & \\
Cte & 4,18 & 0,32 & 13,02 & 0,000 & --- \\
Poder formal & 0,02 & 0,08 & 0,31 & 0,759 & 0,02 \\
Poder informal & $-0,40$ & 0,30 & $-1,35$ & 0,179 & $-0,30$ \\
Oportunidades & $-0,18$ & 0,07 & $-2,64$ & 0,009 & $-0,15$ \\
Información & 0,15 & 0,28 & 0,55 & 0,584 & 0,12 \\
Apoyo & $-0,02$ & 0,07 & $-0,27$ & 0,791 & $-0,02$ \\
Recursos & $-0,34$ & 0,08 & $-4,16$ & 0,000 & $-0,26$ \\
M3 & & & & & --- \\
Cte & 3,42 & 0,44 & 7,66 & 0,000 & 0,01 \\
Poder formal & 0,01 & 0,10 & 0,14 & 0,892 & 0,03 \\
Poder informal & 0,05 & 0,30 & 0,14 & 0,890 & $-0,22$ \\
Oportunidades & $-0,27$ & 0,08 & $-3,36$ & 0,001 & $-0,20$ \\
Información & $-0,27$ & 0,30 & $-0,88$ & 0,380 & $-0,02$ \\
Apoyo & $-0,02$ & 0,07 & $-0,28$ & 0,777 & 0,22 \\
Recursos & $-0,30$ & 0,09 & $-3,23$ & 0,001 & 0,29 \\
Vulnerabilidad al estrés & 0,68 & 0,14 & 5,03 & 0,000 &
\end{tabular}

da del core burnout fue de 20.27\%, F(6,290) = $12.29, \mathrm{p}<0,001$. Estas cuatro variables explicaron un $6.6 \%$ de varianza adicional, cambio en $R^{2}$ $=.06, \mathrm{~F}(4,290)=5.98$, tras controlar el efecto del poder formal e informal. Acceso a oportunidades y acceso a recursos aparecieron como predictores significativos, con valores beta de -.15 $(p=0,009)$ y -.26 $(\mathrm{p}<0,001)$, respectivamente.

Finalmente, en el tercer paso (M3) se añadió la variable vulnerabilidad al estrés como variable independiente. La varianza total explicada en este último modelo fue del $32.50 \%, \mathrm{~F}(7,209)=14.37$, $\mathrm{p}<0,001$. La vulnerabilidad al estrés explicó un $13.3 \%$ de varianza adicional, cambio en $R^{2}=.12$, $\mathrm{F}(1,209)=20.66, \mathrm{p}<0,001$, tras controlar el efecto de las dimensiones del empowerment estructural. En este último modelo aparecieron como predictores significativos del core burnout el acceso a oportunidades (beta $=-.22, \mathrm{p}=0,001$ ), el acceso a recursos (beta $=-.22, \mathrm{p}=0,001)$ y la vulnerabilidad al estrés $($ beta $=.31, \mathrm{p}<0.001)$.

\section{Discusión}

La falta de recursos económicos en los hospitales portugueses y las mayores exigencias laborales hacen que el colectivo de enfermería sea muy vulnerable a las experiencias de estrés y burnout. En este sentido, es necesario el análisis de los elementos organizacionales (i.e., empowerment estructural) e individuales (i.e., vulnerabilidad al estrés) que puede prevenir o contribuir a la aparición del síndrome de "quemarse" en el trabajo. El objetivo de este estudio fue comprobar el papel predictor del empowerment estructural y la vulnerabilidad al estrés sobre el core burnout en una muestra de profesionales enfermeros de Portugal. Los resultados mostraron la relación negativa entre empowerment estructural y burnout, como plantea el modelo de Kanter ${ }^{4}$, así como el papel de la vulnerabilidad al estrés como predictor del estrés en el trabajo.

Los resultados del estudio apoyan la validez del modelo de Kanter y su aplicación en los contextos profesionales de salud en Portugal, específicamente en los profesionales de enfermería. Numerosos estudios realizados por Laschinger y colaboradores usando el modelo de Kanter han mostrado la relación entre elevados niveles de empowerment estructural y menores niveles de core burnout en diferentes muestras de distintos países $^{3,7,11,12}$. Los resultados obtenidos son coherentes con estas investigaciones y apoyan la relación entre factores sociales y organizacionales en el trabajo y bienestar: los elementos estructurales 
y el poder en las organizaciones son importantes condicionantes de la salud y el burnout ${ }^{4,5,7,9}$. Además, los resultados dan un mayor apoyo a la aplicación del modelo del empowerment estructural a la gestión de las unidades de salud.

En este sentido, los resultados muestran la relación existente entre las dimensiones del empowerment estructural y el core burnout. El acceso a información, apoyo, recursos y oportunidades se relacionaron negativamente con el síndrome de "quemarse" en el trabajo. Estos resultados son consistentes con los observados en otros estudios realizados por Cho et al. ${ }^{26} \mathrm{y}$ Laschinger et al. ${ }^{7}$, donde se mostró una fuerte y negativa correlación entre empowerment estructural y burnout. Según Kanter ${ }^{4}$, las percepciones de los profesionales de enfermería sobre su entorno de trabajo juegan un papel relevante en la experiencia de burnout.

Relativamente a la vulnerabilidad al estrés, se registró una relación positiva entre esta variable y la experiencia de burnout. Resultados parecidos fueron registrados en los estudios realizados por Ottati y Freitas ${ }^{19}$, Myhren et al. ${ }^{18}$, Lima et al. ${ }^{15}$ y Hudek-Knezevic et al. ${ }^{2}$. Cuando las personas parecen ser sensibles al estrés, las probabilidades de experimentar estrés y burnout aumentan. La vulnerabilidad afectaría a las estrategias de afrontamiento de los profesionales, creando expectativas negativas sobre su desempeño y el entorno laboral. Si los profesionales de enfermería reaccionan exageradamente (elevada vulnerabilidad) cuando aparecen demandas estresantes en el trabajo, sus capacidades de afrontamiento y su auto-eficacia se ven reducidas afectando igualmente a su rendimiento y creando un círculo vicioso: más estrés, peor afrontamiento, peor desempeño y mayor estrés. Si estos niveles de estrés se hacen frecuentes, es muy probable que aparezca el síndrome de "quemarse en el trabajo".

Los resultados de los modelos de regresión indican que el acceso a recursos, acceso a oportunidades y la vulnerabilidad al estrés fueron los predictores más intensos del burnout, en línea con otras investigaciones realizadas ${ }^{3,5,6}$. El papel predictor del acceso a recursos adquiere sentido en el contexto de crisis y recortes económicos sufridos en las unidades de salud en Portugal en los últimos años. La falta de financiamiento en los hospitales es percibido por los profesionales como falta de recursos que impide realizar adecuadamente el trabajo, a la par que la escasez de los mismos genera estrés y burnout. Como resultado, la falta de recursos se convierte en un importante predictor del core burnout.
Por otro lado y conforme a la teoría de Kanter ${ }^{4}$, la falta de oportunidades para el desarrollo profesional dificulta a los profesionales el aprendizaje de las competencias necesarias para ser eficaces en el trabajo. Los profesionales que se perciben con apenas competencias básicas difícilmente afrontan adecuadamente las demandas y exigencias del día a día, sufriendo elevados niveles de estrés que pueden desembocar en burnout $3,5,7,27,28$.

No obstante, la vulnerabilidad al estrés se ha mostrado como el predictor más fuerte del core burnout. Como se ha indicado anteriormente, individuos vulnerables al estrés pueden sentirse excesivamente saturados cuando afrontan demandas laborales cotidianas que el resto de colegas consideran normales ${ }^{2,15,18,19}$. Estos profesionales especialmente vulnerables perciben que sus recursos personales y/o laborales no son suficientes para afrontar las exigencias y demandas del puesto, de forma que se sienten incapaces de gestionar la situación y las tareas, experimentando elevados niveles de ansiedad y burnout. En resumen, los resultados del estudio muestran la necesidad de actuar sobre las características de la organización, especialmente sobre el acceso a recursos y a oportunidades de desarrollo profesional, así como plantear intervenciones centradas en el tratamiento de la vulnerabilidad del individuo al estrés, utilizando para ello técnicas de relajación en combinación con técnicas cognitivas y comportamentales.

El presente estudio posee una serie de limitaciones que deben tenerse en cuenta. En primer lugar, el diseño transversal y correlacional no permite establecer relaciones de causalidad entre las variables. No obstante, el modelo de regresión utilizado plantea la existencia de variables independientes y dependientes, ya que la revisión de la literatura y la teoría de Kanter ${ }^{4}$ indica que es posible establecer esta relación entre el empowerment estructural, la vulnerabilidad al estrés y el core burnout. Por otro lado, el diseño de encuestas es sensible a determinados sesgos, como el sesgo de deseabilidad en las respuestas dadas o la tendencia en las respuestas de aquellas personas que voluntariamente colaboraron en el estudio ${ }^{29}$. Otra limitación relativa el diseño transversal se relaciona con la presencia de terceras variables que puedan afectar a las relaciones entre las variables del estudio, como por ejemplo la autoeficacia o la resiliencia de los participantes.

Finalmente, con el aumento de las exigencias en las unidades de salud y la falta de recursos debido a la crisis económica, las organizaciones de 
salud deben adoptar estrategias proactivas para la gestión de sus recursos humanos. Este contexto es especialmente estresante para los profesionales sanitarios, como es el caso del personal de enfermería. En consecuencia, el estudio de los elementos organizacionales e individuales que inciden sobre el estrés y el burnout se vuelve vital para las organizaciones.

El empowerment estructural se presenta como una herramienta esencial para la prevención e intervención sobre las características organizacionales asociadas al estrés y el burnout. Planes de formación y de desarrollo profesional mejorarían la percepción por parte de los profesionales de mayores oportunidades para aprender nuevos conocimientos y competencias necesarios para ofrecer cuidados de calidad, así como la participación en la toma de decisiones sobre la organización del trabajo. En el mismo sentido, las direcciones de las unidades de salud deben realizar esfuerzos por gestionar adecuadamente los recursos disponibles: la ausencia de los mismos es interpretada como un obstáculo para la realización de un servicio de calidad.

Por otro lado, la vulnerabilidad al estrés plantea la necesidad de tratamientos orientados a la gestión del estrés entre los profesionales de enfermería, tales como técnicas de relajamiento o de meditación, o el entrenamiento en estrategias de afrontamiento o coping mediante técnicas cognitivo-comportamentales. Igualmente necesarias serían acciones de promoción de la salud y del bienestar basadas en la práctica de actividades deportivas y de gestión saludable del tiempo libre.

\section{Colaboradores}

A Orgambídez-Ramos, Y Borrego-Alés y C Ruiz-Frutos han contribuido intelectualmente en el trabajo, reúnen las condiciones de autoría y han aprobado la versión final del mismo. 


\section{Referencias}

1. Davies A, Wong C, Laschinger HKS. Nurses' participation in personal knowledge transfer: The role of leader-member exchange (LMX) and structural empowerment. J Nurs Manag 2011; 19(5):632-643.

2. Hudek-Knezevic J, Maglica B, Krapiç N. Personality, organizational stress, and attitudes toward work as prospective predictors of professional burnout in hospital nurses. Croat Med J 2011; 52(4):538-549.

3. Lautizi M, Laschinger HKS, Ravazzolo S. Workplace empowerment, job satisfaction, and job stress among Italian mental health nurses: An exploratory study. $J$ Nurs Manag 2009; 17(4):446-452.

4. Kanter RM. Men and women of the corporation. $2^{\mathrm{a}}$ ed. New York: Basic Books; 1993.

5. Laschinger HKS, Finegan J, Shamian J, Wilk P. A longitudinal analysis of the impact of workplace empowerment on work satisfaction. Journal of Organizational Behavior. 2004; 25(4):527-545.

6. Laschinger HKS, Wilk P, Cho J, Greco P. Empowerment, engagement, and perceived effectiveness in nursing work environments: Does experience matter? J Nurs Manag 2009; 17(5):636-646.

7. Laschinger HKS, Wong C, Grau A. Authentic leadership, empowerment, and burnout: A comparison in new graduates and experienced nurses. J Nurs Manag 2013; 21(3):541-552.

8. Mendoza-Sierra MI, Orgambídez-Ramos A, Borrego-Alés Y, Gonçalves G, Santos J. Translation into Spanish of the Conditions for Work Effectiveness Questionnaire (CWEQ-II). Universitas Psicológica 2014; 13(3):15-25.

9. Laschinger HKS, Finegan J, Shamian J, Wilk P. Impact of structural and psychological empowerment on job strain in nursing work settings: Expanding Kanter's model. J Nurs Adm 2001; 31(5):260-272.

10. Laschinger HKS, Finegan J. Using empowerment to build trust and respect in the workplace: A strategy for addressing the nursing shortage. Nurs Econ 2005; 23(1):6-14.

11. Gilbert S, Laschinger HKS, Leiter M. The mediating effects of burnout on the relationship between structural empowerment and organizational citizenship behaviours. J Nurs Manag 2010; 18(3):339-348.

12. Sarmiento TP, Laschinger HKS, Iwasiw C. Nurse educators' workplace empowerment, burnout, and job satisfaction: testing Kanter's theory. J Adv Nurs 2004; 46(2):134-143.

13. George JM, Brief AP. Personality and workrelated distress. In: Schneirand B, Smith B, editors. Personality and organizations. Mahwah: Erlbaum; 2004. p. 193-219.

14. Fernández-García MV, Zárate-Grajales RA, Bautista-Paredes L et al. Síndrome de burnout y enfermería. Evidencia para la práctica. Revista de Enfermería del Instituto Mexicano del Seguro Social 2012; 20(1):45-53.

15. Lima RAS, Souza AI, Galindo RH, Feliciano KVO Vulnerabilidade ao burnout entre médicos de hospital público do Recife. Cien Saude Colet 2013; 18(4):10511058.

16. Sharma A, Sharp DM, Walker LG, Monson JR. Stress and burnout among colorectal surgeons and colorectal nurse specialist working in the National Health Service. Int J Colorectal Dis 2008; 10(4):397-406.
17. Burisch MA. A longitudinal study of burnout: The relative importance of dispositions and experiences. Work Stress 2002; 16(1):1-17.

18. Myhren H, Ekeberg O, Stokland O. Job satisfaction and Burnout among Intensive Care Unit Nurses and Physicians. Crit Care Res Pract 2013; 2013:786176.

19. Ottati F, Freitas V. Evaluation of quality of life and vulnerability to stress in hospital context. Estudos Interdisciplinares em Psicologia. 2013;4 (1):15-29.

20. Serra A. Developing a instrument for assessing stress vulnerability: 23QVS. Psiquiatria Clínica 2000; 21(4): 297-308.

21. Wong C, Laschinger HKS. Authentic leadership, performance, and job satisfaction: The mediating role of empowerment. J Adv Nurs 2013; 69(4):947-959.

22. Montero I, León OG. A guide for naming research studies in Psychology. Int J Health Psychol 2007; 7(3): 847-862.

23. Melo BT, Gomes AR, Cruz JF. Desenvolvimento e adaptação de um instrumento de avaliação psicológica do burnout para os profissionais de psicologia [Development and Adaptation of a psychological instrument for assessing burnout among psychologists]. En: Soares A, Araújo S, Caires S, organizadores. Avaliação Psicológica: Formas e Contextos. Braga: APPORT; 1999. p. 134-156.

24. Maslach C, Jackson M. The measurement of experienced burnout. Journal of Occupational Behaviour 1981; 2:99-113.

25. Orgambídez-Ramos A, Gonçalves G, Santos J, Mendoza-Sierra MI, Borrego-Alés Y. Empowering employees: A Portuguese adaptation of the conditions for work effectiveness questionnaire (CEWQ-II). Psicologia Revista da Associação Portuguesa de Psicologia 2015; 29(1):1-10.

26. Cho J, Laschinger HKS, Wong C. Workplace empowerment, work engagement and organizational commitment of bew graduate nurses. Nurs Leadersh (Tor Ont). 2006; 19(3):43-60.

27. Armstrong K, Laschinger HKS, Wong C. Workplace empowerment and magnet hospital characteristics as predictos of patient safety climate. J Nurs Care Qual 2009; 24(1):55-62.

28. Seibert SE, Wang G, Courtright SH. Antecedents and consequences of psychological and team empowerment in organizations: A meta-analytic review. Journal of Applied Psychology 2011; 96(5):981-1003.

29. Navas JM. Métodos, diseños y técnicas de investigación psicológica. Madrid: UNED; 2002.

Artigo apresentado em 15/08/2015

Aprovado em 17/12/2015

Versão final apresentada em 19/12/2015 